\title{
(3) Die virtuelle Rekonstruktion der Bibliothek der Kartause Buxheim: buxheimlibrary.org
}

\author{
William Whobrey
}

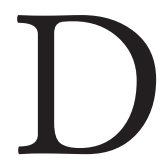
ie Gemeinde Buxheim bei Memmingen im Südwesten Deutschlands wird seit mehr als 600 Jahren von der dort 1402 gegründeten Reichskartause dominiert. Die Geschichte dieses Klosters ist vor allem wegen dessen Bibliothek und deren eventuellen Schicksals bekannt. ${ }^{1}$ Die Kartause wurde 1803 säkularisiert und ging I8Io mit all ihren Einrichtungen in den Besitz der Grafen Waldbott von Bassenheim über. Graf Hugo, der zweite Eigentümer aus diesem Hause, verschleuderte die Buxheimer Kulturgüter, sodass er Konkurs anmelden und den größten Teil des beweglichen Besitzes verkaufen musste, einschließlich der Bibliothek, die auf insgesamt etwa 20.000 Bände geschätzt wird. Zusammen mit Hunderten von spätmittelalterlichen Handschriften und Inkunabeln wurde 1883 die Bibliothek in München versteigert. Dieser Vorgang ist in einem Auktionskatalog von rund 4500 Einträgen dokumentiert. ${ }^{2}$ Der Großteil der Bibliothek gelangte in den Besitz des Münchner Antiquars Ludwig Rosenthal (I840-I928), der selbst unweit der Kartause aufgewachsen war und sogar die Schule in Buxheim besuchte. Im Laufe der nächsten Jahrzehnte, bis heute, wurden Buxheimer Bücher auf dem Antiquariatmarkt angeboten und bereicherten somit die Sammlungen vieler Bibliotheken in Europa und Nordamerika sowie die vieler Privatsammler.

Die Geschichte dieser in alle vier Himmelsrichtungen zerstreute Bibliothek faszinierte mich bereits, als ich junger Assistant Professor für Germanistik an der Yale University war. Ab 1998 begann ich ein Projekt mit dem Ziel, das damals noch deutlich begrenztere Medium des Internets zu nutzen, um die Handschriftenbestände Buxheims als virtuelle Bibliothek zu rekonstruieren und wieder zu vereinigen. ${ }^{3}$ Einige Hilfsmittel bieten sich für den Ansatz eines solchen Unternehmens an, ${ }^{4}$ aber der einzige erhaltene Buxheimer Handschriftenkatalog ist eine einfache Buchliste aus dem späten I5. Jahrhundert mit etwa 2I8 Artikeln. ${ }^{5}$ Mit einer ersten Liste von Handschriften und deren Standorten begann ich, einige dieser Bibliotheken zu besuchen, vor allem die an amerikanischen Universitäten sowie die großen Staatsbibliotheken in München und Berlin. Aber auch kurze Besuche z. B. in Kopenhagen, Stockholm und St. Petersburg boten die Möglichkeit, einzelne Bände aus Buxheim zu sichten. Ein Besuch in Buxheim im Jahr 1998 und dann wieder 2002 für eine Tagung zum 600. Jahrestag der Gründung der Kartause stellte die persönliche Verbindung zu dem Ort her, der meine Forschung seitdem angetrieben hat. Das Jubiläumsjahr 2002 veranlasste mich auch, eine Ausstellung in der Beinecke Rare Book and Manuscript Library der Yale University zu veranstalten, die rund 20 Bücher ihrer eigenen Sammlung aus Buxheim präsentierte.

Zwar konnte mein Projekt seit 200I einen $\mathrm{Ka}^{-}$ talog der bis dahin identifizierten Handschriften im Internet präsentieren, jedoch blieb viel zu tun. Als meine Karriere eine neue Richtung einschlug, wurde mein Buxheim-Projekt aufgeschoben. Ich hatte weiterhin großes Interesse an der Arbeit, verfügte aber nicht mehr über die dafür benötigten zeitlichen Ressourcen. Im Jahr 2013 veröffentlichte das Buxheimer Kartaushausenmuseum einen Katalog seiner eigenen Bestände, die im Laufe der Jahre auf rund I.00o frühneuzeitliche 
Bände angewachsen waren, auf seiner Website im pdf-Format. ${ }^{6}$ Diesen Katalog erstellte Frau Stephanie Bilmayer-Frank in Zusammenarbeit mit ihrem wissenschaftlichen Berater Prof. Dr. Johannes Hoyer. Meine Pensionierung im Jahr 2017 machte es mir schließlich möglich, zur Arbeit mit der Buxheimer Bibliothek (buxheimlibrary.org) zurückzukehren. Seitdem wurde eine neue private Website erstellt, die die originalen, aber nun auch aktualisierten Handschriftenkataloge enthält, zusammen mit vielen anderen Hilfsmitteln und Informationen.

Das primäre Ziel des Projektes ist nach wie vor, das Wissen über die aktuellen Verwahrungsorte von Buxheimer Handschriften zu sichern und zu erweitern.

Die Buxheimer Bände sind leicht identifizierbar (siehe Abb. I-7). Einige haben am Rücken noch ein Signaturetikett aus dem späten I8. bzw. frühen 19. Jahrhundert, das für Handschriften aus einer einfachen roten Nummer (schwarz für postmittelalterliche Handschriften) besteht, gedruckte Bücher haben eine rote oder schwarze Signatur mit einem Buchstaben und einer Nummer. Besitzvermerke innerhalb des Bandes umfassen verschiedene Stempel, z. B. mit dem Wappen der Kartause oder den Waldbott-Bassenheim'schen Initialen, sowie handschriftliche Vermerke mit Variationen von „Cartusiae Buxiae“.

Bis heute konnten 468 ehemalige Handschriften mit einem von 64 aktuellen Standorten identifiziert werden. Die folgenden Daten können dies einigermaßen kontextualisieren. Der Auktionskatalog von 1883 listete 452 Handschriften auf, aber wir wissen, dass viele Handschriften nicht in diesem Katalog aufgeführt waren. Anhand der noch erhaltenen Buxheimer Signaturen glauben wir, dass es insgesamt wahrscheinlich mehr als 700 Handschriften gab, mit etwa 500 davon aus dem I5. Jahrhundert oder früher. Ludwig Rosenthals eigene Antiquariatskataloge aus den Jahren I884 und 1885 führen 427 unikale Buxheimer Handschriften auf, von denen nur 89 mit Einträgen im
Auktionskatalog übereinstimmen. Somit wären insgesamt etwa 790 individuelle Handschriften in diesen drei Katalogen allein aufgeführt. Aus diesem Handschriftencensus geht hervor, dass noch etwa 300 ehemalige Buxheimer Handschriften aufzuspüren wären.

Die Suche geht weiter. Mit neuen Hilfsmitteln wie Bibliotheks- und Antiquariatskatalogen, die jetzt digital im Internet verfügbar sind, sowie leistungsstarken Suchfunktionen und spezialisierten Websites, ${ }^{7}$ werden fast wöchentlich neue Funde gemacht. Ein Beispiel aus meiner eigenen geographischen Umgebung ist besonders interessant. Adolph Sutro (I830-I898) war ein deutscher Einwanderer, der Mitte des 19. Jahrhunderts in die Vereinigten Staaten kam. Er erwarb ein Vermögen in den westlichen Silberminen und ließ sich später in San Francisco nieder. Als einer der reichsten Männer der Stadt erwarb er Land, Immobilien und eine der damals größten Privatbibliotheken in den Vereinigten Staaten. Reisen nach Europa und Ankäufe bei Auktionen erweiterten seine Bestände an mittelalterlichen Handschriften und Inkunabeln erheblich. So erwarb er auch bei der Buxheimer Auktion 1883 eine unbestimmte Zahl von Büchern. Im Laufe der Jahre blieben diese Bände größtenteils in einem Lagerhaus in der Innenstadt von San Francisco, bis zu jenem schicksalhaften Tag im Jahr 1906, als die Stadt von einem Erdbeben heimgesucht wurde. Der nachfolgende Feuersturm zerstörte fast die ganze Innenstadt, einschließlich eines Großteils von Sutros noch unkatalogisierter Bibliothek. Was gerettet wurde, verblieb schließlich beim Staat von Kalifornien. Die Sutro-Sammlung ist derzeit Teil der California State Library auf dem Campus der San Francisco State University. Es gab Hinweise darauf, dass noch einige Buxheimer Handschriften in der Sutro-Bibliothek existierten, ${ }^{8}$ aber die Sammlung ist immer noch nicht vollständig katalogisiert und die Buxheimer Handschriften galten als verschollen. Mit Hilfe von Diana Kohnke, Bibliothekarin an der Staatsbibliothek, konnte 
ich die beiden von de Ricci aufgelisteten Handschriften wieder identifizieren und eine weitere, bisher unbekannte Buxheimer Handschrift in der Sammlung aufspüren. In ähnlicher Weise warten sicherlich weitere solche Entdeckungen, ob mit Hilfe von Online-Ressourcen oder Recherchen vor Ort.

Es gibt noch viel zu tun. Die Inkunabel-Sammlung in Buxheim umfasste möglicherweise I.000 Bände oder mehr, die noch katalogisiert werden müssten. Das vorrangige Ziel meines Forschungsprojekts bleibt jedoch die Suche nach und Lokalisierung weiterer Buxheimer Handschriften. Dies geschieht durch fortwährende kodikologische und bibliographische Detektivarbeit, sowie durch ein wachsendes Netz von Experten, die über den Verbleib noch nicht identifizierter Handschriften Bescheid wissen könnten. Im antiquarischen Bereich sowie in privaten Sammlungen sind sicherlich noch Entdeckungen zu machen, wie die aktuellen eBay- und Abe-Buchverkäufe belegen, sowie in verschiedenen weniger gut dokumentierten Bibliotheken, darunter z. B. noch aktive Kartausen sowie die Grande Chartreuse selbst. Angesichts der Unterstützung dieses Netzwerks bleibt zu hoffen, dass in den kommenden Monaten und Jahren noch viele weitere Entdeckungen von Buxheimer Handschriften gemacht werden können.

\section{Kontakt}

William Whobrey

400 Mar Vista Dr., Nr. 17 · Monterey · CA $93940 \cdot$ USA

E-Mail: william.whobreyg@gmail.com
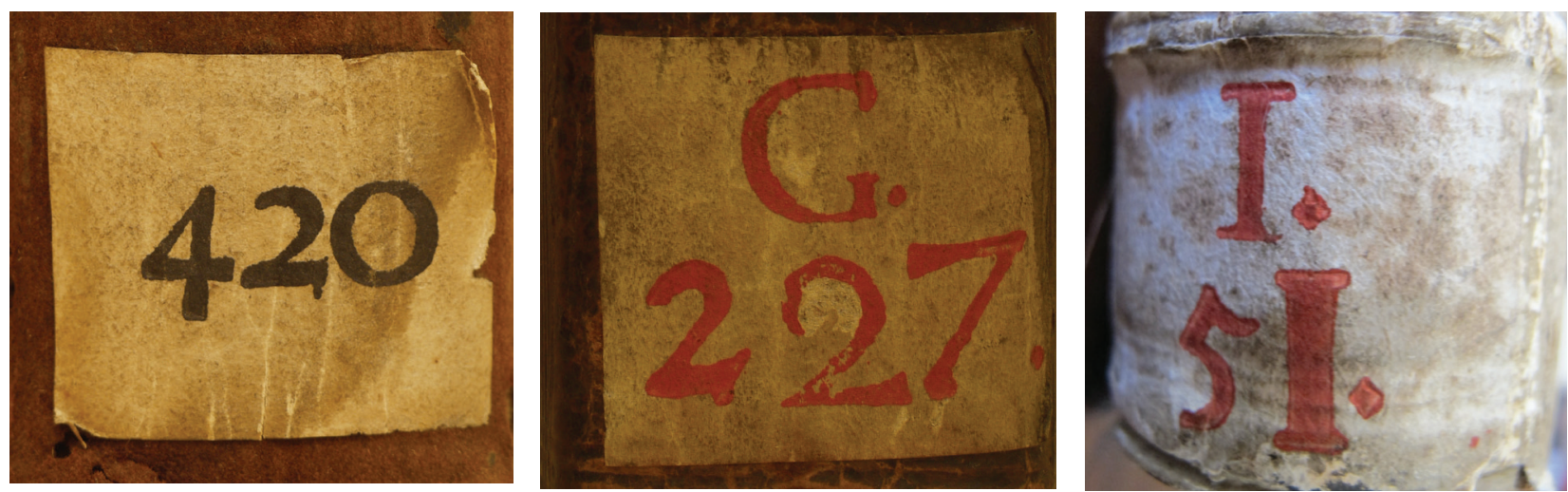

Abb. I-3: Signaturetiketten in rot oder schwarz, mit oder ohne Buchstaben, spätes I8. oder frühes I9. Jahrhundert 

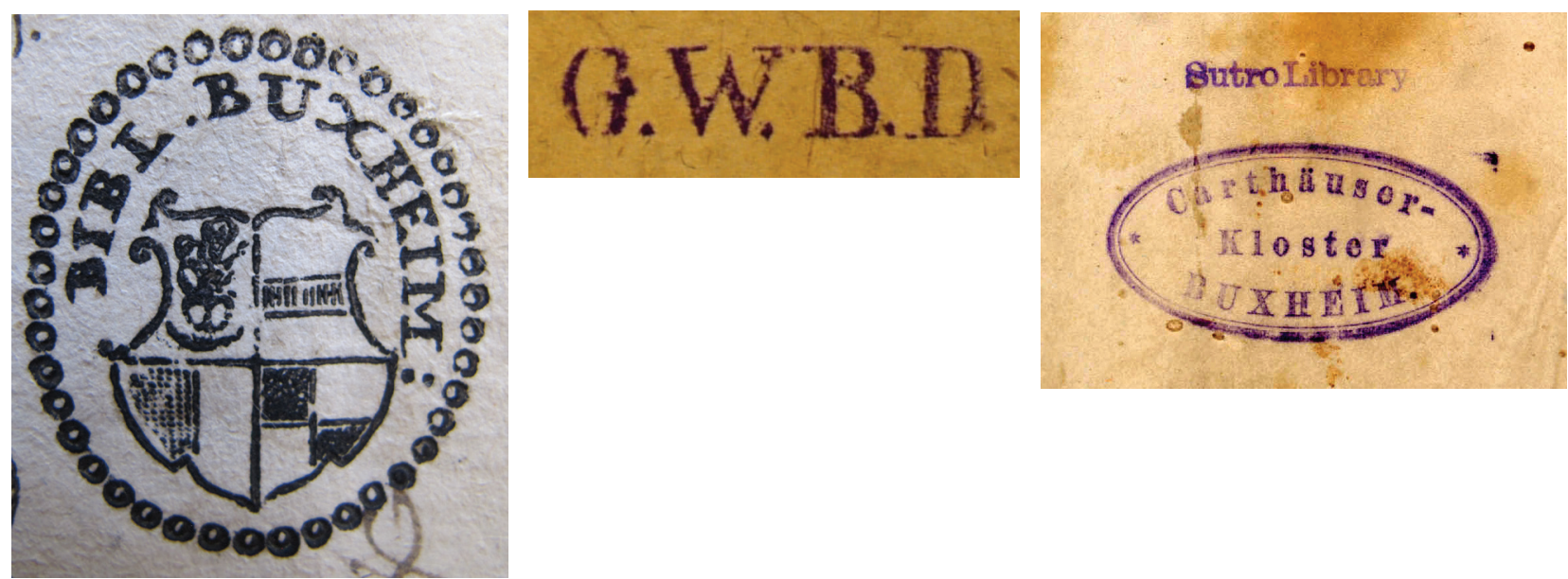

Abb. 4-6: Exlibris-Stempel: 4. Bibl. Buxheim (Kartausenbibliothek, I8. Jh.); 5. G.W.B.D (Gräflich Waldbott-Bassenheim'sche Dominialverwaltung, 19. Jh.); 6. Adolph Sutro Bibliothek, San Francisco (für die 1883 ersteigerten Bände angefertigt). Verbleib: verschollen, wahrscheinlich 1906 vernichtet

\section{Cartufic Buxia Cartusio Buxtreim

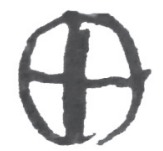

Abb. 7: Zwei typische Besitzvermerke von vielen Variationen des 16. und 17. Jahrhunderts: Cartusiae Buxiae, Cartusiae Buxheim; meist mit dem grafischen Zeichen Kreuz im Zirkel (o. Carth. Zeichen: Stat crux dum volvitur orbis)

\section{Anmerkungen}

I Zur allgemeinen Geschichte der Reichskartause Buxheim siehe Friedrich Stöhlker, Die Kartause Buxheim I402I803, 4 Bde., Heimatdienst Buxheim I974-1978; sowie Friedrich Stöhlker, Die Kartause Buxheim I402-I803/I2: Der Personalschematismus II I554-I8ı2, 3 Bde., Salzburg 1987. Zur Bibliothek siehe Wolfram Sexauer, Frühneuhochdeutsche Schriften in Kartäuserbibliotheken, Frankfurt a. M. 1978; Volker Honemann, The Buxheim Collection and its Dispersal, in: Renaissance Studies 9,2 (1995), S. ı66-ı88.

2 Bibliothek des ehemaligen Carthäuser-Klosters und Gräflich Waldbott-Bassenheimschen Schlosses Buxheim, Carl Förster'sche Kunstauction, München 1883.

3 Dies war einer der frühesten Versuche dieser Art. Klaus Graf, Virtuelle Rekonstruktion historischer Klosterbibliotheken mit Digitalisaten, I5.I2.20I2 (<https://ordensgeschichte.hypotheses.org/I5IO> Zugriff 6.I.202I).

4 Paul Ruf (Hg.), Mittelalterliche Bibliothekskataloge Deutschlands und der Schweiz. Bd. 3,r: Bistum Augsburg. München, I932. Nachdruck I970, S. 8I-IOI; Sigrid Krämer (Hg.), Handschriftenerbe des deutschen Mittelalters, Mittelalterliche Bibiothekskataloge Deutschlands und der Schweiz: Ergänzungsband. 3 Bde., München I989, S. I3I-I43.

5 Berlin, Staatsbibliothek, Ms. lat. qu. 372.

6 <https://www.kartause-buxheim.de/Bibliothek.php> (Zugriff 6.I.202I).

7 Z. B. die Plattformen ,Handschriftenportal‘, ,Handschriftencensus‘, ,e-Codices', ,Manuscripta Mediaevalia', ,Medieval Digital Resources‘ u.v.m.

8 Seymour de Ricci und W. J. Wilson (Hg.), Census of Medieval and Renaissance Manuscripts in the United States and Canada, 3 Bde., New York 1935-1940. 